\title{
The Tightly Super 3-Extra Connectivity and Diagnosability of Locally Twisted Cubes
}

\author{
Mujiangshan Wang1, Yunxia Ren², Yuqing Lin1, Shiying Wang² \\ ${ }^{1}$ School of Electrical Engineering and Computer Science, The University of Newcastle, Newcastle, NSW, Australia \\ ${ }^{2}$ School of Mathematics and Information Science, Henan Normal University, Xinxiang, Henan, China \\ Email: wangshiying@htu.edu.cn,shiying@sxu.edu.cn
}

How to cite this paper: Wang, M., Ren, Y.X., Lin, Y.Q. and Wang, S.Y. (2017) The Tightly Super 3-Extra Connectivity and Diagnosability of Locally Twisted Cubes. American Journal of Computational Mathematics, 7, 127-144.

https://doi.org/10.4236/ajcm.2017.72011

Received: March 20, 2017

Accepted: June 5, 2017

Published: June 8, 2017

Copyright $\odot 2017$ by authors and Scientific Research Publishing Inc. This work is licensed under the Creative Commons Attribution International License (CC BY 4.0).

http://creativecommons.org/licenses/by/4.0/

\begin{abstract}
Diagnosability of a multiprocessor system $G$ is one important measure of the reliability of interconnection networks. In 2016, Zhang et al. proposed the $g$-extra diagnosability of $G$, which restrains that every component of $G-S$ has at least $(g+1)$ vertices. The locally twisted cube $L T Q_{n}$ is applied widely. In this paper, we show that $L T Q_{n}$ is tightly $(4 n-9)$ super 3-extra connected for $n \geq 6$ and the 3-extra diagnosability of $L T Q_{n}$ under the PMC model and $\mathrm{MM}^{*}$ model is $4 n-6$ for $n \geq 5$ and $n \geq 7$, respectively.
\end{abstract}

\section{Keywords}

Interconnection Network, Combinatorics, Diagnosability

\section{Introduction}

At present, semiconductor technology has been widely applied in various fields of large-scale computer systems. But processors or communication links failures of a multiprocessor system give our live a lot of troubles. How to find out the faulty processors accurately and timely becomes the primary problem when the system is in operation. The diagnosis of the system is the process of identifying the faulty processors from the fault-free ones.

There are two well-known diagnosis models, one is the PMC diagnosis model, introduced by Preparata et al. [1] and the other is the MM model, proposed by Maeng and Malek [2]. In the PMC model, any two neighbor processors can test each other. In the MM model, to diagnose a system, we can compare their responses after a node sends the same task to its two neighbors. Sengupta and Dahbura [3] suggested a further modification of the MM model, called the $\mathrm{MM}^{*}$ model, in which each node must test another two neighbors.

In 1996 , the $g$-extra connectivity $\tilde{\kappa}^{(g)}(G)$ of an interconnection network $G$ 
was introduced by Fàbrega and Fiol [4]. The $g$-extra connectivity $\tilde{\kappa}^{(g)}(G)$ of an interconnection network $G$ has been widely studied [4]-[13].

In 2012, Peng et al. [14] proposed a measure for faulty diagnosis of the system, namely, the g-good-neighbor diagnosability, which restrains every fault-free node containing at least $g$ fault-free neighbors. In [14], they studied the $g$-goodneighbor diagnosability of the $n$-dimensional hypercube under the PMC model. In 2016, Wang and Han [15] studied the g-good-neighbor diagnosability of the n-dimensional hypercube under the $\mathrm{MM}^{*}$ model. In 2016, Zhang et al. [16] proposed the $g$-extra diagnosability of the system, which restrains that every component of $G-S$ has at least $(g+1)$ vertices and showed the $g$-extra diagnosability of hypercubes under the PMC model and $\mathrm{MM}^{*}$ model. Ren et al. [17] studied the tightly super 2-extra connectivity and 2-extra diagnosability of locally twisted cubes $L T Q_{n}$. In 2016, Wang et al. [18] studied the 2-extra diagnosability of the bubble-sort star graph $B S_{n}$ under the PMC model and $\mathrm{MM}^{*}$ model. In 2017, Wang and Yang [19] studied the 2-good-neighbor (2extra) diagnosability of alternating group graph networks under the PMC model and $\mathrm{MM}^{*}$ model.

In this paper, we show that $L T Q_{n}$ is tightly $(4 n-9)$ super 3-extra connected for $n \geq 6$ and the 3-extra diagnosability of $L T Q_{n}$ under the PMC model and $\mathrm{MM}^{*}$ model is $4 n-6$ for $n \geq 5$ and $n \geq 7$, respectively.

\section{Preliminaries}

\subsection{Notations}

A multiprocessor system is modeled as an undirected simple graph $G=(V, E)$, whose vertices (nodes) represent processors and edges (links) represent communication links. Suppose that $V^{\prime}$ is a nonempty vertex subset of $V$. The induced subgraph by $V^{\prime}$ in $G$, denoted by $G\left[V^{\prime}\right]$, is a graph, whose vertex set is $V^{\prime}$ and whose edge set consists of all the edges of $G$ with both endpoints in $V^{\prime}$. The degree $d_{G}(v)$ of a vertex $v$ in $G$ is the number of edges incident with $v$. We denote by $\delta(G)$ the minimum degree of vertices of $G$. For any vertex $V$, we define the neighborhood $N_{G}(v)$ of $v$ in $G$ to be the set of vertices adjacent to $v$. $u$ is called a neighbor vertex or a neighbor of $v$ for $u \in N_{G}(v)$. Let $S \subseteq V(G)$. We denote by $N_{G}(S)$ the set $\bigcup_{v \in S} N_{G}(v) \backslash S$. For neighborhoods and degrees, we will usually omit the subscript for the graph when no confusion arises. A graph $G$ is said to be $k$-regular if $d_{G}(v)=k$ for any vertex $v \in V$. A bipartite graph is one whose each edge has one end in subsets of vertex $X$ and one end in subsets of vertex $Y$; such a partition $(X, Y)$ is called a bipartition of the graph. A complete bipartite graph is a simple bipartite graph with bipartition $(X, Y)$ in which each vertex of $X$ is joined to each vertex of $Y$; if $|X|=m$ and $|Y|=n$, such a graph is denoted by $K_{m, n}$. The connectivity $\kappa(G)$ of a connected graph $G$ is the minimum number of vertices whose removal results in a disconnected graph or only one vertex left. Let $F_{1}$ and $F_{2}$ be two distinct subsets of $V$, and let the symmetric difference $F_{1} \Delta F_{2}=\left(F_{1} \backslash F_{2}\right) \cup\left(F_{2} \backslash F_{1}\right)$. For graph-theoretical terminology and notation not defined here we follow [20]. 
Let $G=(V, E)$ be a connected graph. A faulty set $F \subseteq V$ is called a $g$ good-neighbor faulty set if $|N(v) \cap(V \backslash F)| \geq g$ for every vertex $v$ in $V \backslash F$. A $g$-good-neighbor cut of $G$ is a $g$-good-neighbor faulty set $F$ such that $G-F$ is disconnected. The minimum cardinality of $g$-good-neighbor cuts is said to be the $g$-good-neighbor connectivity of $G$, denoted by $\kappa^{(g)}(G)$. A faulty set $F \subseteq V$ is called a $g$-extra faulty set if every component of $G-F$ has at least $(g+1)$ vertices. A $g$-extra cut of $G$ is a $g$-extra faulty set $F$ such that $G-F$ is disconnected. The minimum cardinality of $g$-extra cuts is said to be the $g$-extra connectivity of $G$, denoted by $\tilde{\kappa}^{(g)}(G)$.

Proposition 1. ([21]) Let $G$ be a g-extra and g-good-neighbor connected graph. Then $\tilde{\kappa}^{(g)}(G) \leq \kappa^{(g)}(G)$.

Proposition 2. ([21]) Let $G$ be a 1-good-neighbor connected graph. Then $\kappa^{(1)}(G)=\tilde{\kappa}^{(1)}(G)$.

\subsection{Definitions and Propositions}

Definition 3. ([22] [23] [24] [25]) A system $G$ is said to be t-diagnosable if all faulty processors can be identified without replacement, provided that the number of faults presented does not exceed t. The diagnosability of $G$ is the maximum value of $t$ such that $G$ is $t$-diagnosable.

For the PMC model and $\mathrm{MM}^{*}$ model, we follow [26]. Under the PMC model, to diagnose a system $G=(V(G), E(G))$, two adjacent nodes in $G$ are capable to perform tests on each other. For two adjacent nodes $u$ and $v$ in $V(G)$, the test performed by $u$ on $v$ is represented by the ordered pair $(u, v)$. The outcome of a test $(u, v)$ is 1 (resp. 0$)$ if $u$ evaluate $v$ as faulty (resp. fault-free). We assume that the testing result is reliable (resp. unreliable) if the node $u$ is faultfree(resp. faulty). A test assignment $T$ for $G$ is a collection of tests for every adjacent pair of vertices. The collection of all test results for a test assignment $T$ is called a syndrome. For a given syndrome $\sigma$, a subset of vertices $F \subseteq V(G)$ is said to be consistent with $\sigma$ if syndrome $\sigma$ can be produced from the situation that, for any $(u, v) \in L$ such that $u \in V \backslash F, \sigma(u, v)=1$ if and only if $v \in F$. Let $\sigma(F)$ denote the set of all syndromes which $F$ is consistent with. Under the PMC model, two distinct sets $F_{1}$ and $F_{2}$ in $V(G)$ are said to be indistinguishable if $\sigma\left(F_{1}\right) \cap \sigma\left(F_{2}\right) \neq \varnothing$, otherwise, $F_{1}$ and $F_{2}$ are said to be distinguishable.

Similar to the PMC model, we can define a subset of vertices $F \subseteq V(G)$ is consistent with a given syndrome $\sigma^{*}$ and two distinct sets $F_{1}$ and $F_{2}$ in $V(G)$ are indistinguishable (resp. distinguishable) under the $\mathrm{MM}^{*}$ model.

In a system $G=(V, E)$, a faulty set $F \subseteq V$ is called a $g$-extra faulty set if every component of $G-F$ has more than $g$ nodes. $G$ is $g$-extra $t$-diagnosable if and only if for each pair of distinct faulty $g$-extra vertex subsets $F_{1}, F_{2} \subseteq V(G)$ such that $\left|F_{i}\right| \leq t, F_{1}$ and $F_{2}$ are distinguishable. The $g$-extra diagnosability of $G$, denoted by $\tilde{t}_{g}(G)$, is the maximum value of $t$ such that $G$ is $g$-extra $t$ diagnosable.

Proposition 4. [18] For any given system $G, \tilde{t}_{g}(G) \leq \tilde{t}_{g^{\prime}}(G)$ if $g \leq g^{\prime}$. 
For an integer $n \geq 1$, a binary string of length $n$ is denoted by $u_{1} u_{2} \cdots u_{n}$, where $u_{i} \in\{0,1\}$ for any integer $i \in\{1,2, \cdots, n\}$. The $n$-dimensional locally twisted cube, denoted by $L T Q_{n}$, is an $n$-regular graph of $2^{n}$ vertices and $n 2^{n-1}$ edges, which can be recursively defined as follows [27].

Definition 5. ([27]) For $n \geq 2$, an $n$-dimensional locally twisted cube, denoted by $L T Q_{n}$, is defined recursively as follows.

1) $L T Q_{2}$ is a graph consisting of four nodes labeled with $00,01,10$ and 11 , respectively, connected by four edges $\{00,01\},\{01,11\},\{11,10\}$ and $\{10,00\}$.

2) For $n \geq 3, L T Q_{n}$ is built from two disjoint copies of $L T Q_{n-1}$ according to the following steps. Let $0 L T Q_{n-1}$ denote the graph obtained from one copy of $L T Q_{n-1}$ by prefixing the label of each node with 0 . Let $1 L T Q_{n-1}$ denote the graph obtained from the other copy of $L T Q_{n-1}$ by prefixing the label of each node with 1 . Connect each node $0 u_{2} u_{3} \cdots u_{n}$ of $0 L T Q_{n-1}$ to the node $1\left(u_{2}+u_{n}\right) u_{3} \cdots u_{n}$ of $1 L T Q_{n-1}$ with an edge, where "+" represents the modulo 2 addition.

The edges whose end vertices in different $i L T Q_{n-1} s$ are called to be crossedges. Figures 1-3 show four examples of locally twisted cubes. The locally twisted cube can also be equivalently defined in the following non-recursive fashion.

Definition 6. ([27]) For $n \geq 2$, the n-dimensional locally twisted cube, denoted by $L T Q_{n}$, is a graph with $\{0,1\}^{n}$ as the node set. Two nodes $u_{1} u_{2} \cdots u_{n}$ and $v_{1} v_{2} \cdots v_{n}$ of $L T Q_{n}$ are adjacent if and only if either one of the following conditions are satisfied.

1) $u_{i}=\overline{v_{i}}$ and $u_{i+1}=\left(v_{i+1}+v_{n}\right)(\bmod 2)$ for some $1 \leq i \leq n-2, n \geq 3$ and $u_{j}=v_{j}$ for all the remaining bits;
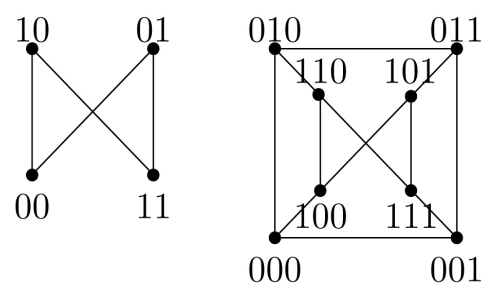

Figure 1. $L T Q_{2}$ and $L T Q_{3}$.

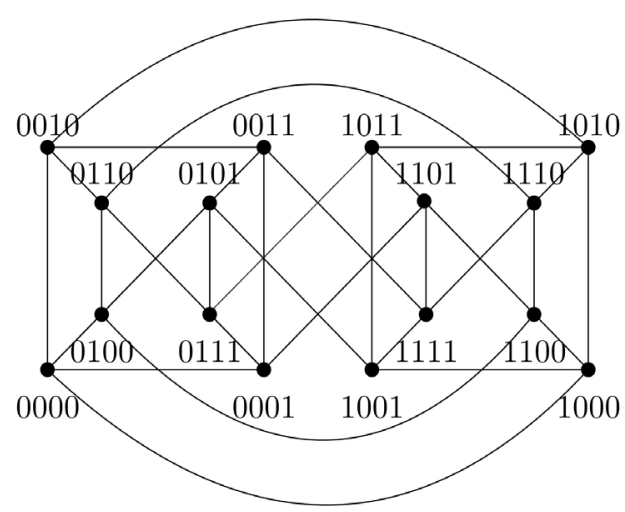

Figure 2. $L T Q_{4}$. 


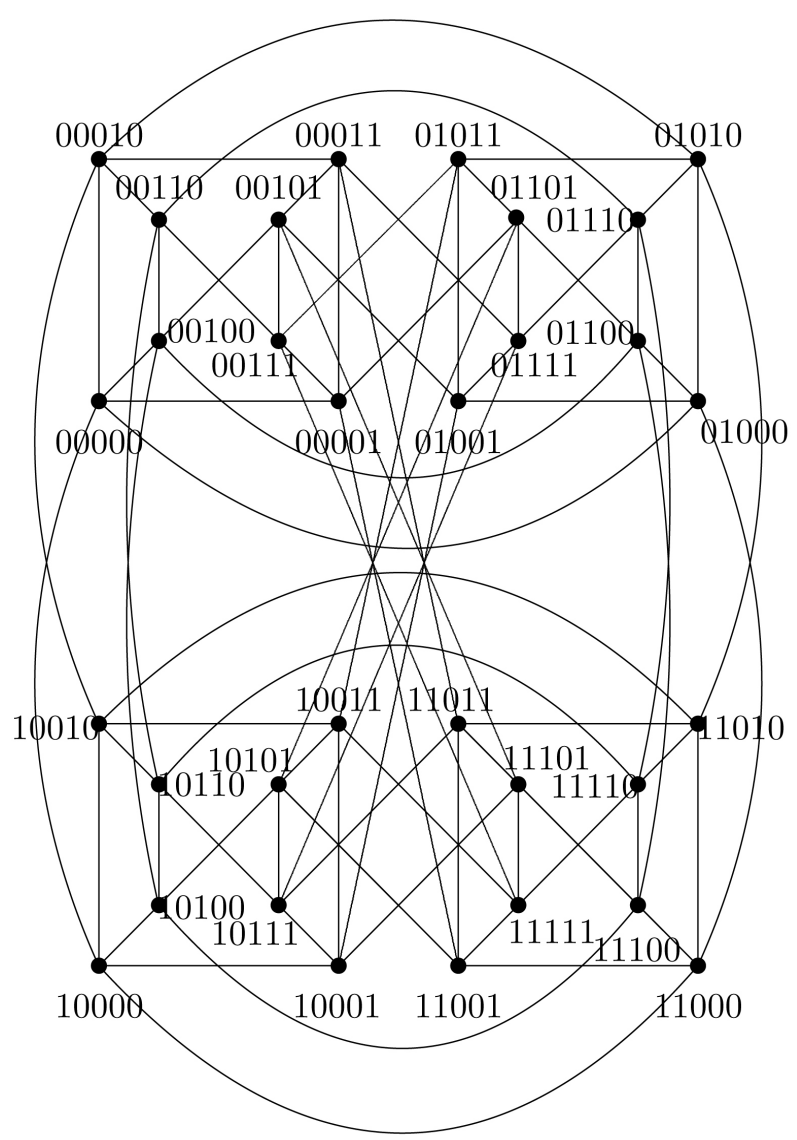

Figure 3. $L T Q_{5}$.

2) $u_{i}=\overline{v_{i}}$ for $i \in\{n-1, n\}, n \geq 2$ and $u_{j}=v_{j}$ for all the remaining bits.

Proposition 7. ([28]) Let $L T Q_{n}$ be the locally twisted cube. If two vertices $u, v$ are adjacent, there is no common neighbor vertex of these two vertices, i.e., $|N(u) \cap N(v)|=0$. If two vertices $u, v$ are not adjacent, there are at most two common neighbor vertices of these two vertices, i.e., $|N(u) \cap N(v)| \leq 2$.

\section{The Connectivity of Locally Twisted Cubes}

Lemma 1. ([27]) Let $L T Q_{n}$ be the locally twisted cube. Then $\kappa\left(L T Q_{n}\right)=n$.

Lemma 2. ([29]) Let $L T Q_{n}$ be the locally twisted cube, and let $S \subseteq V\left(L T Q_{n}\right)$ and $n \geq 3$. If $L T Q_{n}-S$ is disconnected and $n \leq|S| \leq 2 n-3$, then $L T Q_{n}-S$ has exactly two components, one is trivial and the other is nontrivial.

Lemma 3. ([17]) Let $L T Q_{n}$ be the locally twisted cube. Then all cross-edges of $L T Q_{n}$ is a perfect matching.

Lemma 4. ([30]) Let $L T Q_{n}$ be the locally twisted cube. Then $\kappa^{(2)}\left(L T Q_{n}\right)=4 n-8$.

Lemma 5. Let $L T Q_{n}$ be the locally twisted cube. If $P=u v w x$ is a 3-path in $L T Q_{n}$ and $u x \notin E\left(L T Q_{n}\right)$ for $n \geq 3,|N(V(P))| \geq 4 n-9$.

Proof. We decompose $L T Q_{n}$ into $0 L T Q_{n-1}$ and $1 L T Q_{n-1}$. Then $0 L T Q_{n-1}$ and $1 L T Q_{n-1}$ are isomorphic to $L T Q_{n-1}$. Without loss of generality, we have the following cases. 
Case 1. $u, x \in V\left(0 L T Q_{n-1}\right)$ and $v, w \in V\left(1 L T Q_{n-1}\right)$.

Since $u \in V\left(0 L T Q_{n-1}\right), v \in V\left(1 L T Q_{n-1}\right)$ and $u, v$ are adjacent, by Proposition 7, $u, v$ have no the common neighbor vertex. Similarly, $x, w$ have no the common neighbor vertex and $v, w$ have no the common neighbor vertex. Since $u \in V\left(0 L T Q_{n-1}\right), w \in V\left(1 L T Q_{n-1}\right), u, w$ are not adjacent, $v$ is a common neighbor vertex of $u, w, x \in V\left(0 L T Q_{n-1}\right)$ and $x$ is a neighbor vertex of $w$, by Lemma 3, $|(N(u) \cap N(w)) \backslash\{v\}|=0$. Similarly, $|(N(x) \cap N(v)) \backslash\{w\}|=0$. Since $u$ and $x$ are not adjacent, by proposition 7, $|N(u) \cap N(x)| \leq 2$. Therefore, $|N(V(P))| \geq 2(n-1)+2(n-2)-2=4 n-8$.

Case 2. $u \in V\left(0 L T Q_{n-1}\right)$ and $v, w, x \in V\left(1 L T Q_{n-1}\right)$.

Since $u, v$ are adjacent, by Proposition 7, $|N(u) \cap N(v)|=0$. Similarly, $|N(v) \cap N(w)|=0,|N(x) \cap N(w)|=0$. And since $u \in V\left(0 L T Q_{n-1}\right)$, $w \in V\left(1 L T Q_{n-1}\right), u, w$ are not adjacent and $v$ is the common neighbor vertex of $u$ and $w$, by Lemma 3, $|(N(u) \cap N(w)) \backslash\{v\}| \leq 1$. Since $u, x$ are not adjacent, $u \in V\left(0 L T Q_{n-1}\right), \quad x \in V\left(1 L T Q_{n-1}\right)$, by Lemma 3, $|N(u) \cap N(x)| \leq 1$. Since $w$ is the common neighbor vertex of $V$ and $x$ and $v, x$ are not adjacent, by proposition 7, $|(N(v) \cap N(x)) \backslash\{w\}| \leq 1$. Therefore, $|N(P)| \geq 2(n-1)+2(n-2)-3=4 n-9$.

Case 3. $u, v \in V\left(0 L T Q_{n-1}\right)$ and $w, x \in V\left(1 L T Q_{n-1}\right)$.

Since $u, v$ are adjacent, by Proposition 7, $|N(u) \cap N(v)|=0$. Similarly, $|N(u) \cap N(w)|=0,|N(w) \cap N(x)|=0$. Since $u \in V\left(0 L T Q_{n-1}\right)$, $x \in V\left(1 L T Q_{n-1}\right)$ and $u, x$ are not adjacent, by proposition $7,|N(u) \cap N(x)| \leq 2$. If $|(N(u) \cap N(w)) \backslash\{v\}|=1$, then, by Lemma 3, $|N(u) \cap N(x)| \leq 1$. If $|(N(u) \cap N(w)) \backslash\{v\}|=0$, then, by Lemma 3, $|N(u) \cap N(x)| \leq 2$. Therefore, $N(V(P)) \mid \geq 2(n-1)+2(n-2)-2=4 n-8$.

Case 4. $u, v, w, x \in V\left(1 L T Q_{n-1}\right)$.

This case is clear.

In conclusion, $|N(V(P))| \geq 4 n-9$.

Lemma 6. Let $L T Q_{n}$ be the locally twisted cube. If $L_{T} Q_{n}[\{u, v, w, x\}]$ is isomorphic to $K_{1,3}$ for $n \geq 3$ and $d(u)=3$, then $\left|N\left(V\left(L T Q_{n}[\{u, v, w, x\}]\right)\right)\right| \geq 4 n-9$.

Proof. Since $d(u)=3$ and $\operatorname{LTQ}_{n}[\{u, v, w, x\}]$ is isomorphic to $K_{1,3}$, we have $d(v)=1, d(w)=1$ and $d(x)=1$. Since $v, w$ are not adjacent and $u$ is a common neighbor vertex of $v, w$, by Proposition 7, $|(N(v) \cap N(w)) \backslash\{u\}| \leq 1$. Similarly, $|(N(v) \cap N(x)) \backslash\{u\}| \leq 1,|(N(w) \cap N(x)) \backslash\{u\}| \leq 1$. Therefore, $\left|N\left(V\left(L T Q_{n}[\{u, v, w, x\}]\right)\right)\right| \geq 3(n-1)+(n-3)-3=4 n-9$.

If $\operatorname{LTQ}_{n}[\{u, v, w, x\}]$ is a 4 -cycle, then $\left|N\left(V\left(L T Q_{n}[\{u, v, w, x\}]\right)\right)\right|=4 n-8$. Combining this with Lemmas 5 and 6, we have the following corollary.

Corollary 1. Let $L T Q_{n}$ be the locally twisted cube and let $H$ be a connected subgraph of $L T Q_{n}$. If $|V(H)| \geq 4$, then $|N(V(H))| \geq 4 n-9$.

Lemma 7. Let $A=\{0 \cdots 0001,0 \cdots 0111,0 \cdots 0101,0 \cdots 0100\}$ and let $L T Q_{n}$ be the locally twisted cube with $n \geq 4$. If $F_{1}=N_{L T Q_{n}}(A), F_{2}=F_{1} \cup A$, where $n \geq 4$, then $\left|F_{1}\right|=4 n-9,\left|F_{2}\right|=4 n-5, F_{1}$ is a 3-extra cut of $L T Q_{n}$, $L T Q_{n}-F_{1}$ has two components $L T Q_{n}-F_{2}$ and $\operatorname{LTQ}_{n}[A]$, 
$\left|V\left(L T Q_{n}-F_{2}\right)\right| \geq 4$, and $|A| \geq 4$.

Proof. According to the definition, $L T Q_{n}[A]$ is a 3-path and $|A|=4$. By Lemma 5, $\left|F_{1}\right| \geq 4 n-9$. From Figure 2 and the definition of $L T Q_{n}$, we have that $\left|F_{1}\right|=2(n-3)+2(n-2)-3+4=4 n-9$. Therefore,

$\left|F_{2}\right|=\left|F_{1}\right|+|A|=(4 n-9)+4=4 n-5$. Let $F_{2}^{i}=V\left(i L T Q_{n-1}\right) \cap F_{2}, \quad i \in\{0,1\}$.

To prove $L T Q_{n}-F_{2}$ has two components and $\left|V\left(L T Q_{n}-F_{2}\right)\right| \geq 4$, we have the following discussion.

Claim 1. $L T Q_{n}-F_{2}$ is connected for $n \geq 4$.

The proof is by induction on $n$. For $n=4, A=\{0001,0111,0101,0100\}$, $F_{1}=\{0000,0011,0110,1001,1011,1101,1100\}$. It is easy to see that $L T Q_{4}-F_{2}$ is connected (See Figure 2). When $n=5, A=\{00001,00111,00101,00100\}$, $F_{2}^{1}=\{11001,11110,11111,10100\} \quad$ (See Figure 3). It is clear that $1 L T Q_{n-1}-F_{2}^{1}$ is connected (See Figure 3). We discompose $L T Q_{n}$ into $0 L T Q_{n-1}$ and $1 L T Q_{n-1}$. Assume that $n \geq 6$, the result holds for $L T Q_{n-1}$. Then $0 L T Q_{n-1}-F_{2}^{0}$ is connected. Note that $A \subseteq V\left(0 L T Q_{n-1}\right)$ and $\left|N(A) \cap V\left(1 L T Q_{n-1}\right)\right|=4$. By Lemma $1,1 L T Q_{n-1}-F_{2}^{1}$ is connected. By inductive hypothesis, $0 L T Q_{n-1}-F_{2}^{0}$ is connected. Since $2^{n-1}>4 n-5$, by Lemma $3, L T Q_{n}-F_{2}$ is connected. The proof of Claim 1 is complete.

By Claim $1, L T Q_{n}-F_{1}$ has two components $L T Q_{n}-F_{2}$ and $L T Q_{n}[A]$ for $n \geq 4$. Then $\left|V\left(L T Q_{n}-F_{2}\right)\right|=2^{n}-(4 n-5) \geq 4$ for $n \geq 4$. And since $|A|=4$, $F_{1}$ is a 3-extra cut of $L T Q_{n}$.

Lemma 8. ([17]) Let $L T Q_{n}(n \geq 4)$ be the locally twisted cube. If $|F| \leq 3 n-6$, then $L T Q_{n}-F$ satisfies one of the following conditions.

1) $L T Q_{n}-F$ has three components, two of which are isolated vertices;

2) $L T Q_{n}-F$ has two components, one of which is an isolated vertex;

3) $L T Q_{n}-F$ has two components, one of which is a $K_{2}$;

4) $L T Q_{n}-F$ is connected.

Theorem 8. ([31]) Let $L T Q_{n}$ be the locally twisted cube. Then $\tilde{\kappa}^{(3)}\left(L T Q_{n}\right)=4 n-9$ for $n \geq 4$.

Lemma 9. Let $L T Q_{n}$ be the locally twisted cube. If $|F|=10$ for $n=5$, then $L T Q_{5}-F$ satisfies one of the following conditions.

1) $L T Q_{5}-F$ has four components, three of which are isolated vertices;

2) $L T Q_{5}-F$ has three components, one of which is isolated vertices and one of which is a $K_{2}$;

3) $L T Q_{5}-F$ has three components, two of which are isolated vertices;

4) $L T Q_{5}-F$ has two components, one of which is a path of length two;

5) $L T Q_{5}-F$ has two components, one of which is an isolated vertex;

6) $L T Q_{5}-F$ has two components, one of which is a $K_{2}$;

7) $L T Q_{5}-F$ is connected.

Proof. We decompose $L T Q_{5}$ into $0 L T Q_{4}$ and $1 L T Q_{4}$. Then $0 L T Q_{4}$ and $1 L T Q_{4}$ are isomorphic to $L T Q_{4}$. Suppose that $F_{i}=F \cap V\left(i L T Q_{4}\right), i \in\{0,1\}$. Without loss of generality, let $\left|F_{0}\right| \geq\left|F_{1}\right|$. And since $|F|=10,5 \leq\left|F_{0}\right| \leq 10$, $0 \leq\left|F_{1}\right| \leq 5$. Let $C_{i}$ be the maximum component of $i L T Q_{4}-F_{i}, i \in\{0,1\}$. We consider the following cases. 
Case 1. $\left|F_{0}\right|=5$.

Since $\left|F_{0}\right|=5$ and $|F|=10,\left|F_{1}\right|=10-5=5$. By Lemmas 1 and 2, both $0 L T Q_{4}-F_{0}$ and $1 L T Q_{4}-F_{1}$ are connected or has two components, one of which is an isolated vertex. Since $2^{5-1}-6-2 \geq 1$, by Lemma 3 ,

$L T Q_{n}\left[V\left(C_{0}\right) \cup V\left(C_{1}\right)\right]$ is connected. Thus, $L T Q_{5}-F$ satisfies one of conditions:

1) $L T Q_{5}-F$ has three components, two of which are isolated vertices;

2) $L T Q_{5}-F$ has two components, one of which is an isolated vertex;

3) $L T Q_{5}-F$ has two components, one of which is a $K_{2}$;

4) $L T Q_{5}-F$ is connected.

Case 2. $\left|F_{0}\right|=6$.

Since $\left|F_{0}\right|=6$ and $|F|=10,\left|F_{1}\right|=10-6=4$. By Lemmas 1 and 2 , $1 L T Q_{4}-F_{1}$ is connected or has two components, one of which is an isolated vertex. Since $\left|F_{0}\right|=6$, by Lemma $8,0 L T Q_{4}-F_{0}$ satisfies one of the following conditions:

1) $0 L T Q_{4}-F_{0}$ has three components, two of which are isolated vertices;

2) $0 L T Q_{4}-F_{0}$ has two components, one of which is an isolated vertex;

3) $0 L T Q_{4}-F_{0}$ has two components, one of which is a $K_{2}$;

4) $0 L T Q_{4}-F_{0}$ is connected.

Then $L T Q_{5}-F$ satisfies one of the conditions (1)-(7).

Case 3. $\left|F_{0}\right| \geq 7$.

Since $\left|F_{0}\right| \geq 7$ and $|F|=10,\left|F_{1}\right| \leq 10-7=3$. By Lemma $1,1 L T Q_{4}-F_{1}$ is connected.

Suppose that $0 L T Q_{4}-F_{0}$ is connected. Since $2^{5-1}-10 \geq 1$, by Lemma 3 , $L T Q_{n}-F$ is connected.

Suppose that $0 L T Q_{4}-F_{0}$ is not connected. Let the components in $0 L T Q_{4}-F_{0}$ be $G_{1}, G_{2}, \cdots, G_{k}$ for $k \geq 2$ and $\left|V\left(G_{1}\right)\right| \leq\left|V\left(G_{2}\right)\right| \leq \cdots \leq\left|V\left(G_{k}\right)\right|$. If $\left|V\left(G_{r}\right)\right| \geq 4(1 \leq r \leq k-1)$, by Lemma 3, $\left|N\left(V\left(G_{r}\right)\right) \cap V\left(1 L T Q_{4}\right)\right| \geq 4$.

Combining this with $\left|F_{1}\right| \leq 3$, we have that $L T Q_{5}\left[V\left(G_{r}\right) \cup V\left(1 L T Q_{4}-F_{1}\right)\right]$ is connected. Therefore, $G_{r}$ is not a component of $L T Q_{5}-F$ for $\left|V\left(G_{r}\right)\right| \geq 4$. Therefore, $L T Q_{5}-F$ is connected. The following we discuss $G_{r}$ is a component of $L T Q_{5}-F$ with $\left|V\left(G_{r}\right)\right| \leq 3(1 \leq r \leq k-1)$.

If $k=5$, by Lemma 3, $\left|N\left(V\left(G_{1}\right)\right) \cup N\left(V\left(G_{2}\right)\right) \cup \cdots \cup N\left(V\left(G_{k-1}\right)\right) \cap V\left(1 L T Q_{4}\right)\right| \geq 4$. Combining this with $\left|F_{1}\right| \leq 3$, there is one $G_{r}(1 \leq r \leq k-1)$ such that

$L T Q_{5}\left[V\left(G_{r}\right) \cup V\left(1 L T Q_{4}-F_{1}\right)\right]$ is connected. Thus, $k \leq 4$. Since $\left|F_{1}\right|=3$, $k \leq 4$, and $\left|V\left(G_{r}\right)\right| \leq 3(1 \leq r \leq k-1), L T Q_{5}-F$ satisfies one of the conditions (1)-(7).

Lemma 10. Let $L T Q_{n}$ be the locally twisted cube. If $3 n-5 \leq|F| \leq 4 n-10$ for $n \geq 5$, then $L T Q_{n}-F$ satisfies one of the following conditions.

1) $L T Q_{n}-F$ has four components, three of which are isolated vertices;

2) $L T Q_{n}-F$ has three components, one of which is isolated vertices and one of which is a $K_{2}$;

3) $L T Q_{n}-F$ has three components, two of which are isolated vertices; 
4) $L T Q_{n}-F$ has two components, one of which is a path of length two;

5) $L T Q_{n}-F$ has two components, one of which is an isolated vertex;

6) $L T Q_{n}-F$ has two components, one of which is a $K_{2}$;

7) $L T Q_{n}-F$ is connected.

Proof. By Lemma 9, the result holds for $n=5$. We proceed by induction on $n$. Assume $n \geq 6$ and the result holds for $L T Q_{n-1}$, i.e., if $3 n-5 \leq|F| \leq 4(n-1)-10=4 n-14$, then $L T Q_{n-1}-F$ satisfies one of the conditions (1)-(7) in Lemma 10. The following we prove $L T Q_{n}-F$ satisfies one of the conditions (1)-(7).

We decompose $L T Q_{n}$ into $0 L T Q_{n-1}$ and $1 L T Q_{n-1}$. Then $0 L T Q_{n-1}$ and $1 L T Q_{n-1}$ are isomorphic to $L T Q_{n-1}$. Suppose that $F_{i}=F \cap V\left(i L T Q_{n-1}\right)$, $i \in\{0,1\}$. Without loss of generality, let $\left|F_{0}\right| \geq\left|F_{1}\right|$. And since $3 n-5 \leq|F| \leq 4 n-10, n \leq\left\lceil\frac{3 n-5}{2}\right\rceil \leq\left|F_{0}\right| \leq 4 n-10, \quad 0 \leq\left|F_{1}\right| \leq\left\lfloor\frac{4 n-10}{2}\right\rfloor \leq 2 n-5$.

Let $C_{i}$ be the maximum component of $i L T Q_{n-1}-F_{i}, i \in\{0,1\}$. We consider the following cases.

Case 1. $n \leq\left|F_{0}\right| \leq 3(n-1)-6=3 n-9$.

Since $\left|F_{0}\right| \geq\left|F_{1}\right|$ and $|F| \leq 4 n-10$, $(4 n-10)-(3 n-9)=n-1 \leq\left|F_{1}\right| \leq\left\lfloor\frac{4 n-10}{2}\right\rfloor=2 n-5$. By Lemmas 1 and 2 ,

$1 L T Q_{n-1}-F_{1}$ is connected or has two components, one of which is an isolated vertex. Since $n \leq\left|F_{0}\right| \leq 3(n-1)-6=3 n-9$, by lemma $8,0 L T Q_{n-1}-F_{0}$ satisfies one of the following conditions: 1) $0 L T Q_{n-1}-F_{0}$ has three components, two of which are isolated vertices; 2) $0 L T Q_{n-1}-F_{0}$ has two components, one of which is an isolated vertex; 3) $0 L T Q_{n-1}-F_{0}$ has two components, one of which is a $K_{2}$; 4) $0 L T Q_{n-1}-F_{0}$ is connected. Since $2^{n-1}-(4 n-10)-3 \geq 1$, by Lemma 3 , $L T Q_{n}\left[V\left(C_{0}\right) \cup V\left(C_{1}\right)\right]$ is connected. Thus, $L T Q_{n}-F$ satisfies one of conditions (1)-(7) in Lemma 10.

Case 2. $3 n-8 \leq\left|F_{0}\right| \leq 4 n-14$.

Since $\left|F_{0}\right| \geq\left|F_{1}\right|$ and $|F| \leq 4 n-10,\left|F_{1}\right| \leq(4 n-10)-(3 n-8)=n-2$. By Lemma $1,1 L T Q_{n-1}-F_{1}$ is connected. Since $3 n-8 \leq\left|F_{0}\right| \leq 4 n-14$, according to inductive hypothesis, $0 L T Q_{n-1}-F_{0}$ satisfies one of the following conditions:

1) $0 L T Q_{n-1}-F_{0}$ has four components, three of which are isolated vertices;

2) $0 L T Q_{n-1}-F_{0}$ has three components, one of which is isolated vertices and one of which is a $K_{2}$;

3) $0 L T Q_{n-1}-F_{0}$ has three components, two of which are isolated vertices;

4) $0 L T Q_{n-1}-F_{0}$ has two components, one of which is a path of length two;

5) $0 L T Q_{n-1}-F_{0}$ has two components, one of which is an isolated vertex;

6) $0 L T Q_{n-1}-F_{0}$ has two components, one of which is a $K_{2}$;

7) $0 L T Q_{n-1}-F_{0}$ is connected.

Thus, $L T Q_{n}-F$ satisfies one of the conditions (1)-(7) in Lemma 10.

Case 3. $4 n-13 \leq\left|F_{0}\right| \leq 4 n-10$.

Since $4 n-13 \leq\left|F_{0}\right| \leq 4 n-10$ and $|F| \leq 4 n-10$, $\left|F_{1}\right| \leq(4 n-10)-(4 n-13)=3$. By Lemma $1,1 L T Q_{n-1}-F_{1}$ is connected. 
Suppose that $0 L T Q_{n-1}-F_{0}$ is connected. Since $2^{n-1}-(4 n-10) \geq 1$, by Lemma $3, L T Q_{n}-F$ is connected.

Suppose that $0 L T Q_{n-1}-F_{0}$ is not connected. Let the components in $0 L T Q_{n-1}-F_{0}$ be $G_{1}, G_{2}, \cdots, G_{k}$ for $k \geq 2$ and $\left|V\left(G_{1}\right)\right| \leq\left|V\left(G_{2}\right)\right| \leq \cdots \leq\left|V\left(G_{k}\right)\right|$. If $\left|V\left(G_{r}\right)\right| \geq 4(1 \leq r \leq k-1)$, by Lemma 3, $\left|N\left(V\left(G_{r}\right)\right) \cap V\left(1 L T Q_{n-1}\right)\right| \geq 4$.

Combining this with $\left|F_{1}\right| \leq(4 n-10)-(4 n-13)=3$, we have that $L T Q_{n}\left[V\left(G_{r}\right) \cup V\left(1 L T Q_{n-1}-F_{1}\right)\right]$ is connected. Therefore, $G_{r}$ is not a component of $L T Q_{n}-F$ for $\left|V\left(G_{r}\right)\right| \geq 4$. Therefore, $L T Q_{n}-F$ is connected. The following we discuss $G_{r}$ is a component of $L T Q_{n}-F$ with $\left|V\left(G_{r}\right)\right| \leq 3(1 \leq r \leq k-1)$.

If $k=5$, by Lemma 3 , $\left|N\left(V\left(G_{1}\right)\right) \cup N\left(V\left(G_{2}\right)\right) \cup \cdots \cup N\left(V\left(G_{k-1}\right)\right) \cap V\left(1 L T Q_{n-1}\right)\right| \geq 4$. Combining this with $\left|F_{1}\right| \leq 3$, there is one $G_{r}(1 \leq r \leq k-1)$ such that $L T Q_{n}\left[V\left(G_{r}\right) \cup V\left(1 L T Q_{n-1}-F_{1}\right)\right]$ is connected. Thus, $k \leq 4$. Since $\left|F_{1}\right| \leq 3$, $\left|V\left(G_{r}\right)\right| \leq 3(1 \leq r \leq k-1)$ and $k \leq 4, L T Q_{n}-F$ satisfies one of the conditions (1)-(7).

A connected graph $G$ is super $g$-extra connected if every minimum $g$-extra cut $F$ of $G$ isolates one connected subgraph of order $g+1$. In addition, if $G-F$ has two components, one of which is the connected subgraph of order $g+1$, then $G$ is tightly $|F|$ super $g$-extra connected.

Theorem 9. Let $L T Q_{n}$ be the locally twisted cube for $n \geq 6$. Then $L T Q_{n}$ is tightly $(4 n-9)$ super 3 -extra connected.

Proof. By Theorem 8, we know for any minimum 3-extra cut $F \subset V\left(L T Q_{n}\right)$, $|F|=4 n-9$. We decompose $L T Q_{n}$ into $0 L T Q_{n-1}$ and $1 L T Q_{n-1}$. Then $0 L T Q_{n-1}$ and $1 L T Q_{n-1}$ are isomorphic to $L T Q_{n-1}$. Suppose that $F_{i}=F \cap V\left(i L T Q_{n-1}\right), i \in\{0,1\}$. Without loss of generality, let $\left|F_{0}\right| \geq\left|F_{1}\right|$. And since $|F|=4 n-9, \quad 2 n-4 \leq\left\lceil\frac{4 n-9}{2}\right\rceil \leq\left|F_{0}\right| \leq 4 n-9, \quad 0 \leq\left|F_{1}\right| \leq\left\lfloor\frac{4 n-9}{2}\right\rfloor \leq 2 n-5$. Let $C_{i}$ be the maximum component of $i L T Q_{n-1}-F_{i}, i \in\{0,1\}$. We consider the following cases.

Case 1. $2 n-4 \leq\left|F_{0}\right| \leq 3(n-1)-6=3 n-9$.

Since $\left|F_{0}\right| \geq\left|F_{1}\right|$ and $|F|=4 n-9,\left|F_{1}\right| \leq 2 n-5$ holds.

By Lemmas 1 and 2, $1 L T Q_{n-1}-F_{1}$ is connected or has two components, one of which is an isolated vertex. Since $2 n-4 \leq\left|F_{0}\right| \leq 3(n-1)-6=3 n-9$, by lemma 8, $0 L T Q_{n-1}-F_{0}$ satisfies one of the following conditions: 1) $0 L T Q_{n-1}-F_{0}$ has three components, two of which are isolated vertices; 2) $0 L T Q_{n-1}-F_{0}$ has two components, one of which is an isolated vertex; 3) $0 L T Q_{n-1}-F_{0}$ has two components, one of which is a $\left.K_{2} ; 4\right) 0 L T Q_{n-1}-F_{0}$ is connected. Since $2^{n-1}-(4 n-9)-3 \geq 1$, by Lemma 3, $L T Q_{n}\left[V\left(C_{0}\right) \cup V\left(C_{1}\right)\right]$ is connected. Then $L T Q_{n}-F$ satisfies one of the following conditions:

1) $L T Q_{n}-F$ has four components, three of which are isolated vertices;

2) $L T Q_{n}-F$ has three components, one of which is isolated vertices and one of which is a $K_{2}$;

3) $L T Q_{n}-F$ has three components, two of which are isolated vertices; 
4) $L T Q_{n}-F$ has two components, one of which is a path of length two;

5) $L T Q_{n}-F$ has two components, one of which is an isolated vertex;

6) $L T Q_{n}-F$ has two components, one of which is a $K_{2}$;

7) $L T Q_{n}-F$ is connected.

Thus, in this case, $F$ is not a minimum 3-extra cut of $L T Q_{n}$, a contradiction.

Case 2. $\left|F_{0}\right|=3 n-8$.

Since $\left|F_{0}\right|=3 n-8$ and $|F|=4 n-9$, we have $\left|F_{1}\right|=(4 n-9)-(3 n-8)=n-1$. By Lemmas 1 and 2, $1 L T Q_{n-1}-F_{1}$ is connected or has two components, one of which is an isolated vertex. Since $\left|F_{0}\right|=3 n-8$, by Lemma $10,0 L T Q_{n-1}-F_{0}$ satisfies one of the following conditions:

1) $0 L T Q_{n-1}-F_{0}$ has four components, three of which are isolated vertices;

2) $0 L T Q_{n-1}-F_{0}$ has three components, one of which is isolated vertices and the other of which is a $K_{2}$;

3) $0 L T Q_{n-1}-F_{0}$ has three components, two of which are isolated vertices;

4) $0 L T Q_{n-1}-F_{0}$ has two components, one of which is a path of length two;

5) $0 L T Q_{n-1}-F_{0}$ has two components, one of which is an isolated vertex;

6) $0 L T Q_{n-1}-F_{0}$ has two components, one of which is a $K_{2}$;

7) $0 L T Q_{n-1}-F_{0}$ is connected.

If $0 L T Q_{n-1}-F_{0}$ satisfies the condition (4), i.e., $0 L T Q_{n-1}-F_{0}$ has two components, one of which is a path of length two, denoted by $P=u v w, 1 L T Q_{n-1}-F_{1}$ has two components, one of which is an isolated vertex $x$, and $|N(x) \cap V(P)|=1$, $\left(N(V(P)) \cap V\left(1 L T Q_{n-1}\right)\right) \backslash\{x\} \subseteq F_{1}$, then, by Lemma $3, L T Q_{n}-F$ has one component which is a 3 -path or a $K_{1,3}$. Since $2^{n-1}-(4 n-9)-3 \geq 1$ for $n \geq 6$, $L T Q_{n}\left[C_{0} \cup C_{1}\right]$ is connected. Thus, $L T Q_{n}-F$ exactly has two components. Then the other component $C$ satisfies $|C|=2^{n}-(4 n-9)-4>4$ for $n \geq 6$. Otherwise, $F$ is not a minimum 3-extra cut of $L T Q_{n}$, a contradiction.

Case 3. $3 n-7 \leq\left|F_{0}\right| \leq 4 n-14$.

Since $\left|F_{0}\right| \geq\left|F_{1}\right|$ and $|F| \leq 4 n-9,\left|F_{1}\right| \leq(4 n-9)-(3 n-7)=n-2$. By Lemma $1,1 L T Q_{n-1}-F_{1}$ is connected. Since $3 n-7 \leq\left|F_{0}\right| \leq 4 n-14$, by Lemma 10 , $0 L T Q_{n-1}-F_{0}$ satisfies one of the following conditions:

1) $0 L T Q_{n-1}-F_{0}$ has four components, three of which are isolated vertices;

2) $0 L T Q_{n-1}-F_{0}$ has three components, one of which is isolated vertices and the other of which is a $K_{2}$;

3) $0 L T Q_{n-1}-F_{0}$ has three components, two of which are isolated vertices;

4) $0 L T Q_{n-1}-F_{0}$ has two components, one of which is a path of length two;

5) $0 L T Q_{n-1}-F_{0}$ has two components, one of which is an isolated vertex;

6) $0 L T Q_{n-1}-F_{0}$ has two components, one of which is a $K_{2}$;

7) $0 L T Q_{n-1}-F_{0}$ is connected.

Thus, $L T Q_{n}-F$ satisfies one of the following conditions:

1) $L T Q_{n}-F$ has four components, three of which are isolated vertices;

2) $L T Q_{n}-F$ has three components, one of which is isolated vertices and one of which is a $K_{2}$;

3) $L T Q_{n}-F$ has three components, two of which are isolated vertices;

4) $L T Q_{n}-F$ has two components, one of which is a path of length two;

5) $L T Q_{n}-F$ has two components, one of which is an isolated vertex; 
6) $L T Q_{n}-F$ has two components, one of which is a $K_{2}$;

7) $L T Q_{n}-F$ is connected.

In this case, $F$ is not a minimum 3-extra cut of $L T Q_{n}$, a contradiction.

Case 4. $\left|F_{0}\right|=4 n-13$.

Since $\left|F_{0}\right|=4 n-13$ and $|F|=4 n-9$ for $n \geq 6$, $\left|F_{1}\right|=(4 n-9)-(4 n-13)=4$. By Lemma $1,1 L T Q_{n-1}-F_{1}$ is connected.

If there exists a 3-path $P$ in $0 L T Q_{n-1}-F_{0}$, then $N(V(P)) \cap V\left(0 L T Q_{n-1}\right) \subseteq F_{0}$. By Corollary 1, $|N(V(P))| \geq 4 n-13=\left|F_{0}\right|$ in $0 L T Q_{n-1}-F_{0}$. Therefore, $N(V(P))=F_{0}$ in $0 L T Q_{n-1}-F_{0}$. Note that $2^{n-1}-(4 n-9)-4 \geq 1$ for $n \geq 6$, by Lemma 3, then $L T Q_{n}\left[V\left(C_{0}\right) \cup V\left(C_{1}\right)\right]$ is connected. Then $L T Q_{n}-F$ just has two components, one of which is a 3-path.

If there exists a component $K_{1,3}$ in $0 L T Q_{n-1}-F_{0}$, then $N_{0 L T Q_{n-1}}\left(V\left(K_{1,3}\right)\right) \subseteq F_{0}$. By Corollary 1, $\left|N\left(V\left(K_{1,3}\right)\right)\right| \geq 4 n-13=\left|F_{0}\right|$ in $0 L T Q_{n-1}-F_{0}$. Therefore, $N\left(V\left(K_{1,3}\right)\right)=F_{0}$ in $0 L T Q_{n-1}-F_{0}$. Note that $2^{n-1}-(4 n-9)-4 \geq 1$ for $n \geq 6$, by Lemma $3, L T Q_{n}-F$ just has two components, one of which is a $K_{1,3}$.

If there exists a 4 -cycle $C$ in $0 L T Q_{n-1}-F_{0}$, then $N_{0 L T Q_{n-1}}(C) \cap V\left(0 L T Q_{n-1}\right) \subseteq F_{0}$. By Proposition 7, $\left|N_{0 L T Q_{n-1}}(V(C))\right| \geq 4(n-1-2)=4 n-12>4 n-13=\left|F_{0}\right|$, a contradiction to $\left|F_{0}\right|=4 n-13$. Therefore, $0 L T Q_{n-1}-F_{0}$ has not a 4-cycle.

Case 5. $4 n-12 \leq\left|F_{0}\right| \leq 4 n-9$.

Since $4 n-12 \leq\left|F_{0}\right| \leq 4 n-9$ and $|F| \leq 4 n-9,\left|F_{1}\right| \leq(4 n-9)-(4 n-12)=3$. By Lemma $1,1 L T Q_{n-1}-F_{1}$ is connected.

Suppose that $0 L T Q_{n-1}-F_{0}$ is connected. Since $2^{n-1}-(4 n-9) \geq 1$, by Lemma 3, $L T Q_{n}-F$ is connected, a contradiction.

Suppose that $0 L T Q_{n-1}-F_{0}$ is not connected. Let the components in $0 L T Q_{n-1}-F_{0}$ be $G_{1}, G_{2}, \cdots, G_{k}$ for $k \geq 2$ and $\left|V\left(G_{1}\right)\right| \leq\left|V\left(G_{2}\right)\right| \leq \cdots \leq\left|V\left(G_{k}\right)\right|$. If $\left|V\left(G_{r}\right)\right| \geq 4(1 \leq r \leq k-1)$, by Lemma 3, $\left|N\left(V\left(G_{r}\right)\right) \cap V\left(1 L T Q_{n-1}\right)\right| \geq 4$. If $k \geq 5$, by Lemma 3 , $\left|N\left(V\left(G_{1}\right)\right) \cup N\left(V\left(G_{2}\right)\right) \cup \ldots \cup N\left(V\left(G_{k-1}\right)\right) \cap V\left(1 L T Q_{n-1}\right)\right| \geq 4$. Combining this with $\left|F_{1}\right| \leq(4 n-9)-(4 n-12)=3$, we have that $L T Q_{n}-F$ satisfies one of the following conditions:

1) $L T Q_{n}-F$ has four components, three of which are isolated vertices;

2) $L T Q_{n}-F$ has three components, one of which is isolated vertices and one of which is a $K_{2}$;

3) $L T Q_{n}-F$ has three components, two of which are isolated vertices;

4) $L T Q_{n}-F$ has two components, one of which is a path of length two;

5) $L T Q_{n}-F$ has two components, one of which is an isolated vertex;

6) $L T Q_{n}-F$ has two components, one of which is a $K_{2}$;

7) $L T Q_{n}-F$ is connected.

In this case, $F$ is not a minimum 3-extra cut of $L T Q_{n}$, a contradiction.

\section{The 3-Extra Diagnosability of the Locally Twisted Cube under the PMC Model}

In this section, we shall show the 3-extra diagnosability of locally twisted cubes 
under the PMC model.

Theorem 10. ([16] [22] [26]) A system $G=(V, E)$ is g-extra t-diagnosable under the PMC model if and only if there is an edge $u v \in E$ with $u \in V \backslash\left(F_{1} \cup F_{2}\right)$ and $v \in F_{1} \Delta F_{2}$ for each distinct pair of g-extra faulty subsets $F_{1}$ and $F_{2}$ of $V$ with $\left|F_{1}\right| \leq t$ and $\left|F_{2}\right| \leq t$.

Lemma 11. Let $n \geq 4$. Then the 3-extra diagnosability of the locally twisted cube $L T Q_{n}$ under the PMC model is less than or equal to $4 n-6$, i.e., $\tilde{t}_{3}\left(L T Q_{n}\right) \leq 4 n-6$.

Proof. Let $A$ be defined in Lemma 7, and let $F_{1}=N_{L T Q_{n}}(A)$, $F_{2}=A \cup N_{L T Q_{n}}(A)$. By Lemma 7, $\left|F_{1}\right|=4 n-9,\left|F_{2}\right|=|A|+\left|F_{1}\right|=4 n-5$, $\left|V\left(L T Q_{n}[A]\right)\right| \geq 4$ and $\left|V\left(L T Q_{n}-F_{2}\right)\right| \geq 4, F_{1}$ is a 3-extra cut of $L T Q_{n}$. Therefore, $F_{1}$ and $F_{2}$ are 3-extra faulty sets of $L T Q_{n}$ with $\left|F_{1}\right|=4 n-9$ and $\left|F_{2}\right|=4 n-5$. Since $A=F_{1} \Delta F_{2}$ and $N_{L T Q_{n}}(A)=F_{1} \subset F_{2}$, there is no edge of $L T Q_{n}$ between $V\left(L T Q_{n}\right) \backslash\left(F_{1} \cup F_{2}\right)$ and $F_{1} \Delta F_{2}$. By Theorem 10, we can deduce that $L T Q_{n}$ is not 3-extra $(4 n-5)$-diagnosable under PMC model. Hence, by the definition of 3-extra diagnosability, we conclude that the 3-extra diagnosability of $L T Q_{n}$ is less than $4 n-5$, i.e., $\tilde{t}_{3}\left(L T Q_{n}\right) \leq 4 n-6$.

Lemma 12. Let $n \geq 5$. Then the 3-extra diagnosability of the locally twisted cube $L_{T} Q_{n}$ under the PMC model is more than or equal to $4 n-6$, i.e., $\tilde{t}_{3}\left(L T Q_{n}\right) \geq 4 n-6$.

Proof. By the definition of 3-extra diagnosability, it is sufficient to show that $L T Q_{n}$ is 3-extra $(4 n-6)$-diagnosable. By Theorem 10 , to prove $L T Q_{n}$ is 3extra $(4 n-6)$-diagnosable, it is equivalent to prove that there is an edge $u v \in E\left(L T Q_{n}\right)$ with $u \in V\left(L T Q_{n}\right) \backslash\left(F_{1} \cup F_{2}\right)$ and $v \in F_{1} \Delta F_{2}$ for each distinct pair of 3-extra faulty subsets $F_{1}$ and $F_{2}$ of $V\left(L T Q_{n}\right)$ with $\left|F_{1}\right| \leq 4 n-6$ and $\left|F_{2}\right| \leq 4 n-6$.

Suppose, by way of contradiction, that there are two distinct 3-extra faulty subsets $F_{1}$ and $F_{2}$ of $L T Q_{n}$ with $\left|F_{1}\right| \leq 4 n-6$ and $\left|F_{2}\right| \leq 4 n-6$, but the vertex set pair $\left(F_{1}, F_{2}\right)$ is not satisfied with the condition in Theorem 10, i.e., there are no edges between $V\left(L T Q_{n}\right) \backslash\left(F_{1} \cup F_{2}\right)$ and $F_{1} \Delta F_{2}$. Without loss of generality, assume that $F_{2} \backslash F_{1} \neq \varnothing$.

Assume $V\left(L T Q_{n}\right)=F_{1} \cup F_{2}$. Since $n \geq 5$, we have that $2^{n}=\left|V\left(L T Q_{n}\right)\right|=$ $\left|F_{1} \cup F_{2}\right|=\left|F_{1}\right|+\left|F_{2}\right|-\left|F_{1} \cap F_{2}\right| \leq\left|F_{1}\right|+\left|F_{2}\right| \leq(4 n-6)+(4 n-6)=8 n-12$, a contradiction. Therefore, $V\left(L T Q_{n}\right) \neq F_{1} \cup F_{2}$.

The following we discuss the case when $F_{2} \backslash F_{1} \neq \varnothing$ and $V\left(L T Q_{n}\right) \neq F_{1} \cup F_{2}$. Since there are no edges between $V\left(L T Q_{n}\right) \backslash\left(F_{1} \cup F_{2}\right)$ and $F_{1} \Delta F_{2}$, and $F_{1}$ is a 3-extra faulty set, $L T Q_{n}-F_{1}$ has two parts $L T Q_{n}-F_{1}-F_{2}$ and $L T Q_{n}\left[F_{2} \backslash F_{1}\right]$. Thus, every component $G_{i}$ of $L T Q_{n}-F_{1}-F_{2}$ satisfies $\left|V\left(G_{i}\right)\right| \geq 4$ and every component $C_{i}$ of $L T Q_{n}\left[F_{2} \backslash F_{1}\right]$ satisfies $\left|V\left(C_{i}\right)\right| \geq 4$. Similarly, every component $C_{i}^{\prime}$ of $L T Q_{n}\left[F_{1} \backslash F_{2}\right]$ satisfies $\left|V\left(C_{i}^{\prime}\right)\right| \geq 4$ when $F_{1} \backslash F_{2} \neq \varnothing$. Therefore, $F_{1} \cap F_{2}$ is also a 3-extra faulty set. Since there are no edges between $V\left(L T Q_{n}-F_{1}-F_{2}\right)$ and $F_{1} \Delta F_{2}, F_{1} \cap F_{2}$ is also a 3-extra cut. When $F_{1} \backslash F_{2}=\varnothing, F_{1} \cap F_{2}=F_{1}$ is also a 3-extra faulty set. Since there are no edges between $V\left(L T Q_{n}-F_{1}-F_{2}\right)$ and $F_{1} \Delta F_{2}, F_{1} \cap F_{2}$ is a 3-extra cut. By 
Theorem 8, $\left|F_{1} \cap F_{2}\right| \geq 4 n-9$. Therefore,

$\left|F_{2}\right|=\left|F_{2} \backslash F_{1}\right|+\left|F_{1} \cap F_{2}\right| \geq 4+4 n-9=4 n-5$, which contradicts with that

$\left|F_{2}\right| \leq 4 n-6$. So $L T Q_{n}$ is 3-extra $(4 n-6)$-diagnosable. By the definition of $\tilde{t}_{3}\left(L T Q_{n}\right), \tilde{t}_{3}\left(L T Q_{n}\right) \geq 4 n-6$. The proof is complete.

Combining Lemmas 11 and 12, we have the following theorem.

Theorem 11. Let $n \geq 5$. Then the 3-extra diagnosability of the locally twisted cubes $L_{T}$ under the PMC model is $4 n-6$.

\section{The 3-Extra Diagnosability of the Locally Twisted Cube under the $\mathbf{M M}^{*}$ Model}

Before discussing the 3-extra diagnosability of the locally twisted cube $L T Q_{n}$ under the $\mathrm{MM}^{*}$ model, we first give an existing result.

Theorem 12 ([3] [16] [26]) A system $G=(V, E)$ is g-extra t-diagnosable under the $\mathrm{MM}^{*}$ model if and only if for each distinct pair of g-extra faulty subsets $F_{1}$ and $F_{2}$ of $V$ with $\left|F_{1}\right| \leq t$ and $\left|F_{2}\right| \leq t$ satisfies one of the following conditions.

1) There are two vertices $u, w \in V \backslash\left(F_{1} \cup F_{2}\right)$ and there is a vertex $v \in F_{1} \Delta F_{2}$ such that $u w \in E$ and $v w \in E$.

2) There are two vertices $u, v \in F_{1} \backslash F_{2}$ and there is a vertex $w \in V \backslash\left(F_{1} \cup F_{2}\right)$ such that $u w \in E$ and $v w \in E$.

3) There are two vertices $u, v \in F_{2} \backslash F_{1}$ and there is a vertex $w \in V \backslash\left(F_{1} \cup F_{2}\right)$ such that $u w \in E$ and $v w \in E$.

Lemma 13. Let $n \geq 4$. Then the 3-extra diagnosability of the locally twisted cube $L T Q_{n}$ under the $M M^{*}$ model is less than or equal to $4 n-6$, i.e., $\tilde{t}_{3}\left(L T Q_{n}\right) \leq 4 n-6$.

Proof. Let $A$ be defined in Lemma 7, and let $F_{1}=N_{L T Q_{n}}(A)$, $F_{2}=A \cup N_{L T Q_{n}}(A)$. By Lemma 7, $\left|F_{1}\right|=4 n-9,\left|F_{2}\right|=|A|+\left|F_{1}\right|=4 n-5$, $\left|V\left(L T Q_{n}[A]\right)\right| \geq 4$ and $\left|V\left(L T Q_{n}-F_{2}\right)\right| \geq 4, \quad F_{1}$ is a 3-extra cut of $L T Q_{n}$. Therefore, $F_{1}$ and $F_{2}$ are 3-extra faulty sets of $L T Q_{n}$ with $\left|F_{1}\right|=4 n-9$ and $\left|F_{2}\right|=4 n-5$. Since $A=F_{1} \Delta F_{2}$ and $N_{L T Q_{n}}(A)=F_{1} \subset F_{2}$, there is no edge of $L T Q_{n}$ between $V\left(L T Q_{n}\right) \backslash\left(F_{1} \cup F_{2}\right)$ and $F_{1} \Delta F_{2}$. By Theorem 12, we can deduce that $L T Q_{n}$ is not 3-extra $(4 n-5)$-diagnosable under $\mathrm{MM}^{*}$ model. Hence, by the definition of 3-extra diagnosability, we conclude that the 3-extra diagnosability of $L T Q_{n}$ is less than $4 n-5$, i.e., $\tilde{t}_{3}\left(L T Q_{n}\right) \leq 4 n-6$.

A component of a graph $G$ is odd or even according as it has an odd or even number of vertices. We denote by $o(G)$ the number of odd components of $G$.

Lemma 14. ([20]) A graph $G=(V, E)$ has a perfect matching if and only if $o(G-S) \leq|S|$ for all $S \subseteq V$.

Lemma 15. Let $n \geq 7$. Then the 3-extra diagnosability of the locally twisted cube $L T Q_{n}$ under the $M M^{*}$ model is more than or equal to $4 n-6$, i.e., $\tilde{t}_{3}\left(L T Q_{n}\right) \geq 4 n-6$.

Proof. By the definition of the 3-extra diagnosability, it is sufficient to show that $L T Q_{n}$ is 3-extra $(4 n-6)$-diagnosable.

By Theorem 12, suppose, by way of contradiction, that there are two distinct 
3-extra faulty subsets $F_{1}$ and $F_{2}$ of $L T Q_{n}$ with $\left|F_{1}\right| \leq 4 n-6$ and $\left|F_{2}\right| \leq 4 n-6$, but the vertex set pair $\left(F_{1}, F_{2}\right)$ is not satisfied with any one condition in Theorem 12. Without loss of generality, assume that $F_{2} \backslash F_{1} \neq \varnothing$. Similarly to the discussion on $V\left(L T Q_{n}\right)=F_{1} \cup F_{2}$ in Lemma 12, we can deduce $V\left(L T Q_{n}\right) \neq F_{1} \cup F_{2}$. Therefore, we have the following discussion for $V\left(L T Q_{n}\right) \neq F_{1} \cup F_{2}$.

Claim 1. $L T Q_{n}-F_{1}-F_{2}$ has no isolated vertex.

Suppose, by way of contradiction, that $L T Q_{n}-F_{1}-F_{2}$ has at least one isolated vertex $w$. Since $F_{1}$ is a 3-extra faulty set, there is at least one vertex $u \in F_{2} \backslash F_{1}$ such that $u$ are adjacent to $w$. Since the vertex set pair $\left(F_{1}, F_{2}\right)$ is not satisfied with any one condition in Theorem 12, by the condition (3) of Theorem 12, there is at most one vertex $u \in F_{2} \backslash F_{1}$ such that $u$ is adjacent to $w$. Therefore, there is just a vertex $u$ is adjacent to $w$.

Case 1. $F_{1} \backslash F_{2}=\varnothing$.

If $F_{1} \backslash F_{2}=\varnothing$, then $F_{1} \subseteq F_{2}$. Since $F_{2}$ is a 3-extra faulty set, every component $G_{i}$ of $L T Q_{n}-F_{1}-F_{2}$ has $\left|V\left(G_{i}\right)\right| \geq 4$. Thus, $L T Q_{n}-F_{1}-F_{2}$ has no isolated vertex.

Case 2. $F_{1} \backslash F_{2} \neq \varnothing$.

Similarly, since $F_{1} \backslash F_{2} \neq \varnothing$, by the condition (2) of Theorem 12 and the hypothesis, we can deduce that there is just a vertex $v \in F_{1} \backslash F_{2}$ such that $v$ is adjacent to $w$.

Let $W \subseteq V\left(L T Q_{n}\right) \backslash\left(F_{1} \cup F_{2}\right)$ be the set of isolated vertices in $L T Q_{n}\left[V\left(L T Q_{n}\right) \backslash\left(F_{1} \cup F_{2}\right)\right]$, and $H$ be the induced subgraph by the vertex set $V\left(L T Q_{n}\right) \backslash\left(F_{1} \cup F_{2} \cup W\right)$. Then for any $w \in W$, there are $(n-2)$ neighbors in $F_{1} \cap F_{2}$. By Lemmas 14 and 3, $|W| \leq o\left(L T Q_{n}-\left(F_{1} \cup F_{2}\right)\right) \leq\left|F_{1} \cup F_{2}\right|=$ $\left|F_{1}\right|+\left|F_{2}\right|-\left|F_{1} \cap F_{2}\right| \leq(4 n-6)+(4 n-6)-(n-2)=7 n-10$. Assume $V(H)=\varnothing$. Then $2^{n}=\left|V\left(L T Q_{n}\right)\right|=\left|F_{1} \cup F_{2}\right|+|W|=\left|F_{1}\right|+\left|F_{2}\right|-\left|F_{1} \cap F_{2}\right| \leq(4 n-6)+$ $(4 n-6)-(n-2)+(7 n-10)=14 n-20$, a contradiction to that $n \geq 7$. So $V(H) \neq \varnothing$.

The following we discuss the case when $F_{1} \backslash F_{2} \neq \varnothing, \quad F_{2} \backslash F_{1} \neq \varnothing$ and $V(H) \neq \varnothing$.

Since the vertex set pair $\left(F_{1}, F_{2}\right)$ is not satisfied with the condition (1) of Theorem 12, and there are not isolated vertices in $H$, we induce that there is no edge between $V(H)$ and $F_{1} \Delta F_{2}$. Note that $F_{2} \backslash F_{1} \neq \varnothing$. If $F_{1} \cap F_{2}=\varnothing$, then this is a contradiction to that $L T Q_{n}$ is connected. Therefore, $F_{1} \cap F_{2} \neq \varnothing$. Thus, $F_{1} \cap F_{2}$ is a vertex cut of $L T Q_{n}$. Since $F_{1}$ is a 3-extra faulty set of $L T Q_{n}$, we have that every component $H_{i}$ of $H$ has $\left|V\left(H_{i}\right)\right| \geq 4$ and every component $C_{i}$ of $\operatorname{LTQ}_{n}\left[W \cup\left(F_{2} \backslash F_{1}\right)\right]$ has $\left|V\left(C_{i}\right)\right| \geq 4$. Since $F_{2}$ also is a 3-extra faulty set of $L T Q_{n}$, we have that every component $C_{i}^{\prime}$ of $\operatorname{LTQ}_{n}\left[W \cup\left(F_{1} \backslash F_{2}\right)\right]$ has $\left|V\left(C_{i}^{\prime}\right)\right| \geq 4$. Note that $L T Q_{n}-\left(F_{1} \cap F_{2}\right)$ has two parts: $H$ and $L T Q_{n}\left[W \cup\left(F_{1} \Delta F_{2}\right)\right]$. Let $b_{i} \in V\left(L T Q_{n}\left[W \cup\left(F_{1} \Delta F_{2}\right)\right]\right)$. If $b_{i} \in W$, then $b_{i}$ has two neighbors $u \in V\left(C_{i}\right)$ and $v \in V\left(C_{i}^{\prime}\right)$. Then $b_{i} \in V\left(C_{i} \cup C_{i}^{\prime}\right)$ and $\left|V\left(C_{i} \cup C_{i}^{\prime}\right)\right| \geq 4$. Thus, $F_{1} \cap F_{2}$ is a 3-extra cut of $L T Q_{n}$. By Theorem 8, $\left|F_{1} \cap F_{2}\right| \geq 4 n-9$. Since $\left|V\left(C_{i}\right)\right| \geq 4,\left|F_{2} \backslash F_{1}\right| \geq 3$. Since 
$\left|F_{1} \cap F_{2}\right|=\left|F_{2}\right|-\left|F_{2} \backslash F_{1}\right| \leq(4 n-6)-3=4 n-9$, we have $\left|F_{1} \cap F_{2}\right|=4 n-9$. Then $\left|F_{2} \backslash F_{1}\right|=3$ and $\left|F_{2}\right|=4 n-6$. Similarly, $\left|F_{1} \backslash F_{2}\right|=3,\left|F_{1}\right|=4 n-6$. By Lemma 9, the locally twisted cube $L T Q_{n}$ is tightly $(4 n-9)$ super 3-extra connected, i.e., $L T Q_{n}-\left(F_{1} \cap F_{2}\right)$ has two components, one of which is a subgraph of order 4. Noted that $|W| \leq 7 n-10.2^{n}=\left|V\left(L T Q_{n}\right)\right|=\left|F_{1} \backslash F_{2}\right|+\left|F_{2} \backslash F_{1}\right|+\left|F_{1} \cap F_{2}\right|+$ $|V(H)|+|W| \leq 3+3+(4 n-9)+4+(7 n-10)=11 n-9$, a contradiction to $n \geq 7$. Therefore, $L T Q_{n}-F_{1}-F_{2}$ has no isolated vertex when $F_{1} \backslash F_{2} \neq \varnothing$, $F_{2} \backslash F_{1} \neq \varnothing$ and $V(H) \neq \varnothing$. The proof of Claim 1 is complete.

Let $u \in V\left(L T Q_{n}\right) \backslash\left(F_{1} \cup F_{2}\right)$. By Claim $1, u$ has at least one neighbor vertex in $L T Q_{n}-F_{1}-F_{2}$. Since the vertex set pair $\left(F_{1}, F_{2}\right)$ is not satisfied with any one condition in Theorem 12, by the condition (1) of Theorem 12, for any pair of adjacent vertices $u, w \in V\left(L T Q_{n}\right) \backslash\left(F_{1} \cup F_{2}\right)$, there is no vertex $v \in F_{1} \Delta F_{2}$ such that $u w \in E\left(L T Q_{n}\right)$ and $u v \in E\left(L T Q_{n}\right)$. It follows that $u$ has no neighbor vertex in $F_{1} \Delta F_{2}$. By the arbitrariness of $u$, there is no edge between $V\left(L T Q_{n}\right) \backslash\left(F_{1} \cup F_{2}\right)$ and $F_{1} \Delta F_{2}$. Since $F_{2} \backslash F_{1} \neq \varnothing$ and $F_{1}$ is a 3-extra faulty set, $\left|F_{2} \backslash F_{1}\right| \geq 4$ and $\left|V\left(L T Q_{n}-F_{2}-F_{1}\right)\right| \geq 4$. Since $F_{1}$ also is 3-extra faulty sets, $\left|F_{1} \backslash F_{2}\right| \leq 4$ and $\left|V\left(L T Q_{n}-F_{1}-F_{2}\right)\right| \geq 4$. Then $F_{1} \cap F_{2}$ is a 3extra cut of $L T Q_{n}$. By Theorem 8, we have $\left|F_{1} \cap F_{2}\right| \geq 4 n-9$. Therefore, $\left|F_{2}\right|=\left|F_{2} \backslash F_{1}\right|+\left|F_{1} \cap F_{2}\right| \geq 4+(4 n-9)=4 n-5$, which contradicts $\left|F_{2}\right| \leq 4 n-6$. Therefore, $L T Q_{n}$ is 3 -extra $(4 n-6)$-diagnosable and $\tilde{t}_{3}\left(L T Q_{n}\right) \geq 4 n-6$. The proof is complete.

Combining Lemmas 13 and 15, we have the following theorem.

Theorem 13. Let $n \geq 7$. Then the 3-extra diagnosability of the locally twisted cube $L T Q_{n}$ under the $M M^{*}$ model is $4 n-6$.

\section{Acknowledgements}

This work is supported by the National Science Foundation of China (61370001).

\section{References}

[1] Preparata, F.P., Metze, G. and Chien, R.T. (1967) On the Connection Assignment Problem of Diagnosable Systems. IEEE Transactions on Computers, EC-16, 848854. https://doi.org/10.1109/PGEC.1967.264748

[2] Maeng, J. and Malek, M. (1981) A Comparison Connection Assignment for SelfDiagnosis of Multiprocessor Systems. Proceeding of 11 th International Symposium on Fault-Tolerant Computing, 173-175.

[3] Sengupta, A. and Dahbura, A.T. (1992) On Self-Diagnosable Multiprocessor Systems: Diagnosis by the Comparison Approach. IEEE Transactions on Computers, 41, 1386-1396. https://doi.org/10.1109/12.177309

[4] Fàbrega, J. and Fiol, M.A. (1996) On the Extraconnectivity of Graphs. Discrete Mathematics, 155, 49-57.

[5] Gu, M.-M., Hao, R.-X. and Liu J.-B. (2017) On the Extraconnectivity of k-Ary nCube Networks. International Journal of Computer Mathematics, 94, 95-106. https://doi.org/10.1080/00207160.2015.1091070

[6] Chang, N.-W., Tsai, C.-Y. and Hsieh, S.-Y. (2014) On 3-Extra Connectivity and 3-Extra Edge Connectivity of Folded Hypercubes. IEEE Transactions on Computers, 63, 1593-1599. 
[7] Zhang, M.-M. and Zhou, J.-X. (2015) On g-Extra Connectivity of Folded Hypercubes. Theoretical Computer Science, 593, 146-153.

[8] Hsieh, S.-Y. and Chang, Y.-H. (2012) Extraconnectivity of k-Ary n-Cube Networks. Theoretical Computer Science, 443, 63-69.

[9] Gu, M.-M. and Hao, R.-X. (2014) 3-Extra Connectivity of 3-Ary n-Cube Networks. Information Processing Letters, 114, 486-491.

[10] Lin, R.Z. and Zhang, H.P. (2016) The Restricted Edge-Connectivity and Restricted Connectivity of Augmented $k$-Ary $n$-Cubes. International Journal of Computer Mathematics, 93, 1281-1298. https://doi.org/10.1080/00207160.2015.1067690

[11] Lü, H. (2017) On Extra Connectivity and Extra Edge-Connectivity of Balanced Hypercubes. International Journal of Computer Mathematics, 94, 813-820. https://doi.org/10.1080/00207160.2016.1148813

[12] Hong, W.-S. and Hsieh, S.-Y. (2013) Extra Edge Connectivity of Hypercube-Like Networks. International Journal of Parallel, Emergent and Distributed Systems, 28, 123-133. https://doi.org/10.1080/17445760.2011.650696

[13] Xu, J.M., Wang, J.W. and Wang, W.W. (2010) On Super and Restricted Connectivity of Some Interconnection Networks. Ars Combinatoria, 94, 1-8.

[14] Peng, S.-L., Lin, C.-K., Tan, J.J.M. and Hsu, L.-H. (2012) The g-Good-Neighbor Conditional Diagnosability of Hypercube under PMC Model. Applied Mathematics and Computation, 218, 10406-10412. https://doi.org/10.1016/j.amc.2012.03.092

[15] Wang, S. and Han, W. (2016) The $g$-Good-Neighbor Conditional Diagnosability of $n$-Dimensional Hypercubes under the $\mathrm{MM}^{\star}$ Model. Information Processing Letters, 116, 574-577. https://doi.org/10.1016/j.ipl.2016.04.005

[16] Zhang, S. and Yang, W. (2016) The $g$-Extra Conditional Diagnosability and Sequential $t / k$-Diagnosability of Hypercubes. International Journal of Computer Mathematics, 93, 482-497. https://doi.org/10.1080/00207160.2015.1020796

[17] Ren, Y. and Wang, S. The Tightly Super 2-Extra Connectivity and 2-Extra Diagnosability of Locally Twisted Cubes. Journal of Interconnection Networks (To Appear).

[18] Wang, S., Wang, Z. and Wang, M. (2016) The 2-Extra Connectivity and 2-Extra Diagnosability of Bubble-Sort Star Graph Networks. The Computer Journal, 59, 1839-1856. https://doi.org/10.1093/comjnl/bxw037

[19] Wang, S. and Yang, Y. (2017) The 2-Good-Neighbor (2-Extra) Diagnosability of Alternating Group Graph Networks under the PMC Model and MM* Model. Applied Mathematics and Computation, 305, 241-250. https://doi.org/10.1016/j.amc.2017.02.006

[20] Bondy, J.A. and Murty, U.S.R. (2007) Graph Theory. Springer, New York.

[21] Ren, Y. and Wang, S. (2016) Some Properties of the g-Good-Neighbor ( $g$-Extra) Diagnosability of a Multiprocessor System. American Journal of Computational Mathematics, 6, 259-266. https://doi.org/10.4236/ajcm.2016.63027

[22] Dahbura, A.T. and Masson, G.M. (1984) An $O\left(n^{2.5}\right)$ Fault Identification Algorithm for Diagnosable Systems. IEEE Transactions on Computers, 33, 486-492. https://doi.org/10.1109/TC.1984.1676472

[23] Fan, J. (2002) Diagnosability of Crossed Cubes under the Comparison Diagnosis Model. IEEE Transactions on Parallel and Distributed Systems, 13, 1099-1104. https://doi.org/10.1109/TPDS.2002.1041887

[24] Fan, J., Zhang, S., Jia, X. and Zhang, G. (2009) The Restricted Connectivity of Locally Twisted Cubes. 10th International Symposium on Pervasive Systems, Algorithms, and Networks (ISPAN), Kaohsiung, 14-16 December 2009, 574-578. 
https://doi.org/10.1109/I-SPAN.2009.48

[25] Lai, P.-L., Tan, J.J.M., Chang, C.-P. and Hsu, L.-H. (2005) Conditional Diagnosability Measures for Large Multiprocessor Systems. IEEE Transactions on Computers, 54, 165-175. https://doi.org/10.1109/TC.2005.19

[26] Yuan, J., Liu, A., Ma, X., Liu, X., Qin, X. and Zhang, J. (2015) The g-Good-Neighbor Conditional Diagnosability of $k$-Ary $n$-Cubes under the PMC model and $\mathrm{MM}^{*}$ Model. IEEE Transactions on Parallel and Distributed Systems, 26, 1165-1177. https://doi.org/10.1109/TPDS.2014.2318305

[27] Yang, X., Evans, D.J. and Megson, G.M. (2005) The Locally Twisted Cubes. International Journal of Computer Mathematics, 82, 401-413. https://doi.org/10.1080/0020716042000301752

[28] Ren, Y. and Wang, S. The 1-Good-Neighbor Connectivity and Diagnosability of Locally Twisted Cubes. Chinese Quarterly Journal of Mathematics (To Appear).

[29] Feng, R., Bian, G. and Wang, X. (2011) Conditional Diagnosability of the Locally Twisted Cubes under the PMC Model. Communications and Network, 3, 220-224. https://doi.org/10.4236/cn.2011.34025

[30] Hsieh, S.-Y., Huang, H.-W. and Lee, C.-W. (2016) \{2,3\}-Restricted Connectivity of Locally Twisted Cubes. Theoretical Computer Science, 615, 78-90. https://doi.org/10.1016/j.tcs.2015.11.050

[31] Zhu, Q., Wang, X.-K. and Cheng, G. (2013) Reliability Evaluation of BC Networks. IEEE Transactions on Computers, 62, 2337-2340.

https://doi.org/10.1109/TC.2012.106

\section{Submit or recommend next manuscript to SCIRP and we will provide best} service for you:

Accepting pre-submission inquiries through Email, Facebook, LinkedIn, Twitter, etc. A wide selection of journals (inclusive of 9 subjects, more than 200 journals)

Providing 24-hour high-quality service

User-friendly online submission system

Fair and swift peer-review system

Efficient typesetting and proofreading procedure

Display of the result of downloads and visits, as well as the number of cited articles

Maximum dissemination of your research work

Submit your manuscript at: http://papersubmission.scirp.org/

Or contact ajcm@scirp.org 